\title{
Long noncoding RNA PVT1 promotes breast (1) cancer proliferation and metastasis by binding miR-128-3p and UPF1
}

Shuiyi Liu ${ }^{1,2+}$, Weiqun Chen ${ }^{1,2,3 \dagger}$, Hui Hu ${ }^{1 \dagger}$, Tianzhu Zhang ${ }^{1,4 \dagger}$, Tangwei Wu' ${ }^{1}$, Xiaoyi Li ${ }^{1}$, Yong Li ${ }^{1,5}$, Qinzhi Kong ${ }^{2,6}$, Hongda $L u^{2,6^{*}}$ and Zhongxin $L u^{1,2,3,4^{*}}$

\begin{abstract}
Background: Mounting evidence supports that long noncoding RNAs (IncRNAs) have critical roles during cancer initiation and progression. In this study, we report that the plasmacytoma variant translocation 1 (PVT1) IncRNA is involved in breast cancer progression.

Methods: qRT-PCR and western blot were performed to detect the gene and protein expression. Colony formation would healing and transwell assays were used to detect cell function. Dual-luciferase reporter assay and RNA pull-down experiments were used to examine the mechanisms interaction between molecules. Orthotopic mouse models were established to evaluate the influence of PVT1 on tumor growth and metastasis in vivo.
\end{abstract}

Results: PVT1 is significant upregulated in breast cancer patients' plasma and cell lines. PVT1 promotes breast cancer cell proliferation and metastasis both in vitro and in vivo. Mechanistically, PVT1 upregulates FOXQ1 via miR-128-3p and promotes epithelial-mesenchymal transition. In addition, PVT1 binds to the UPF1 protein, thereby inducing epithelial-mesenchymal transition, proliferation and metastasis in breast cancer cells.

Conclusion: PVT1 may act as an oncogene in breast cancer through binding miR-128-3p and UPF1 and represents a potential target for BC therapeutic development.

Keywords: Plasmacytoma variant translocation 1, Breast cancer, Epithelial-mesenchymal transition, miR-128-3p, Up-frameshift protein 1

\section{Background}

In 2019, breast cancer (BC) is the most prevalent malignancy among women worldwide and is the second leading cause of cancer-associated death in women after lung

\footnotetext{
*Correspondence: phlonda@163.com; Izx71@yahoo.com

†Shuiyi Liu, Weiqun Chen, Hui Hu and Tianzhu Zhang have contributed equally to this work

${ }^{1}$ Department of Medical Laboratory, The Central Hospital of Wuhan, Tongji Medical College, Huazhong University of Science and Technology, 26 Shengli St., Jiangan District, Wuhan 430014, China

${ }^{2}$ Cancer Research Institute of Wuhan, The Central Hospital of Wuhan,

Tongji Medical College, Huazhong University of Science and Technology, Wuhan 430014, China
}

Full list of author information is available at the end of the article and bronchus cancer, according to the American Cancer Society [1]. In recent decades, great improvements have been made in diagnosis, surgery, chemotherapy, and molecular targeted treatment on BC. Yet, the prognosis for Stage IV BC is far from satisfactory because of its heterogeneity and complexity. Therefore, molecular pathogenesis of $\mathrm{BC}$ should be further investigated to identify better targets for therapeutic development.

Long noncoding RNAs (lncRNAs), previously regarded as transcriptional noise, have gradually become a focus of research in various diseases, especially in cancer $[2,3]$. LncRNAs are endogenous cellular molecules with more than 200nt. Lacking an open reading frame, lncRNAs 
have limited protein-coding capacity [4]. However, accumulating evidence has demonstrated that the abnormal lncRNAs expression is involved in a variety of biological and pathological processes, such as cell proliferation, cell apoptosis, cell invasion, cell metastasis, stem cell renewal, drug resistance, and so on $[5,6]$.

The plasmacytoma variant translocation 1 (PVT1) is an oncogenic lncRNA, located at the $8 \mathrm{q} 24$ region, was discovered in murine plasmacytoma in 1985 [7]. PVT1 is upregulated in numerous cancers, functioning as an oncogene in malignant progression $[8,9]$. PVT1 directly interacts with p-STAT3 to activate the STAT3 signaling pathway, thereby promoting angiogenesis in gastric cancer [10]. Recent research has revealed that PVT1 is up regulated in nasopharyngeal carcinoma tissues and its overexpression predicts a poor prognosis for NPC patients. Loss-of-function experiments support that PVT1 regulates cell apoptosis by influencing the DNA damage repair pathway after radiation, suggesting that targeting PVT1 may be a potential strategy for NPC therapy [11]. However, more data are required to elucidate the functions of PVT1 in tumor progression.

In our study, we report that PVT1 is highly expressed both in plasma from $\mathrm{BC}$ patients and $\mathrm{BC}$ cells. In addition, we show that PVT1 promotes $\mathrm{BC}$ proliferation and metastasis both in vitro and vivo. Mechanistically, PVT1 directly binds UPF1 and competitively recruits miR128-3p to upregulate FOXQ1, thereby promoting BC progression.

\section{Materials and methods Clinical specimens}

In this study, blood samples were collected from female patients at the Central Hospital of Wuhan, including $80 \mathrm{BC}$ patients, 70 healthy volunteers, and 70 fibroma patients; for $35 \mathrm{BC}$ patients, paired blood samples (preoperative and postoperative blood in one month after surgery) were collected. None of the patients had received any clinical treatment before sample collection and corresponding tumor specimens were confirmed by histopathological examination. No diseases or injuries were found in the healthy controls. Peripheral blood samples were collected in EDTA-containing tubes and were processed within $4 \mathrm{~h}$ by centrifugation at $1000 \mathrm{~g}$ for 15 min at $4{ }^{\circ} \mathrm{C}$. The supernatant was then transferred into RNAse-free tubes and stored at $-80{ }^{\circ} \mathrm{C}$. This study was approved by the Medical Ethics Committee of The Central Hospital of Wuhan and all human subject research was performed in accordance with institutional, national, and Declaration of Helsinki requirements.

\section{Cell culture}

The human BC cell lines (Hs578t, MCF-7, MDA-MB-231, T47D, Bt549, HCC1806), the noncancerous breast epithelial HBL-100 cells, and the human embryonic kidney (HEK) $293 \mathrm{~T}$ cells were purchased from the American Type Culture Collection (ATCC). Cell culture was performed following the recommendations of ATCC. We confirm the authentication of all cell lines used-the full policy and requirements are available in the instructions to authors.

\section{Plasmids construction and cell transfection}

The pSIF-GFP-miR-128-3p precursor plasmid and the precursor control were gifts from Dr. Yong $\mathrm{Li}$ in Baylor College of Medicine. miR-128-3p inhibitors and miRNA controls were obtained from GenePharma Technology (Shanghai, China). Small interfering RNAs (siRNAs) targeting PVT1 and a negative control were also obtained from GenePharma Technology (Shanghai, China). Three shRNAs targeting human UPF1 and the shRNA negative control sequence were as follows: shUPF1\#1, sense: 5'-GATCCG AGCCACATTGTAAATCAT TTCAAG AGAATGATTTA CAATGTGGCTTTTTTTG-3, antisense: 5'-AATTCAAAAAAAGCCACATTGTAAATC ATTCTCTTGAAATGATTTACAATGTGGCTCG-3'; shUPF1\#2, sense: 5'-GATCCG GCGAGAAGGACTTCA TCATTCAAGAGATGATGAAGTCCTTCTCGCTTT TTTG-3', antisense: 5'-AATTCAAAAAAGCGAGAA GGACTTCATCATCTCTTGAATGATGAA GTCCTT CTCGCCG-3'; shUPF1\#3, sense: 5'-GATCCGGCAGCC ACATTGTAAAT.

CATCAAGAG TGATTTACAATGTGGCTGCTT TTTTG-3', antisense: 5'-AATTCA AAAAAGCAGCCA CATTGTAAATCACTCTTGATGATTTACAATGTG GCTGCCG-3' The shRNA constructs were cloned into Bam HI and Eco RI sites in an RNAi-ready pSIRENRetroQ vector (Clontech, USA). All DNA constructs were confirmed by Sanger sequencing. Transfections were performed using Lipofectamine ${ }^{\circledR}$ LTX and Plus reagent (Invitrogen, USA) according to the manufacturer's instructions.

\section{Colony formation assay}

After transfection, Hs578t or MCF-7 cells were seeded into 12-well plates at 500 cells/well and cultured in DMEM with $10 \% \mathrm{FBS}$ at $37^{\circ} \mathrm{C}$ for 2 weeks. Plates were washed twice with PBS, colonies were then fixed with $4 \%$ paraformaldehyde for $20 \mathrm{~min}$ and stained with $0.1 \%$ crystal violet for $30 \mathrm{~min}$. More than 50 cells were counted as a colony. 


\section{RNA immunoprecipitation (RIP) assay}

RIP was performed using a EZ-Magna RIP kit (Millipore, USA) according to the manufacturer's instructions. In brief, Hs578t or MCF-7 cells were lysed in the complete RIP lysis buffer and then the lysates were incubated with beads-antibody in RIP buffer. Rabbit anti-UPF1 antibody and rabbit IgG control (Millipore, USA) were used. Finally, the co-precipitated RNAs were extracted and detected by qRT-PCR.

\section{Wound healing assay}

Hs578t or MCF-7 cells were seeded in 6-well plates. A wound was made by a sterile $200 \mu \mathrm{l}$ pipette tip $24 \mathrm{~h}$ post transfection. The images $(0 \mathrm{~h})$ were taken using a microscope. After incubation at $37{ }^{\circ} \mathrm{C}$ for $24 \mathrm{~h}$, cells were washed twice with PBS and the wound healing images (24 h) were photographed again.

\section{Transwell assay}

To investigate the capacities of cell invasion, transwell chamber (Corning, USA) was coated with Matrigel (BD Biosciences, USA). Hs578t or MCF-7 cells $\left(5 \times 10^{4}\right)$ were starved in $200 \mu \mathrm{L}$ serum-free medium $24 \mathrm{~h}$ post transfection and then seeded into the top chamber of each insert ( $8 \mu \mathrm{m}$, Corning, USA). The lower chambers were filled with $600 \mu \mathrm{L}$ of complete medium. After cultured at $37{ }^{\circ} \mathrm{C}$ for $24 \mathrm{~h}$, cells migrated to the lower chambers were fixed, stained, and counted.

\section{RNA isolation and qRT-PCR}

RNA was extracted using TRIzol reagent (Invitrogen, USA). cDNA was quantified by SYBR green (Applied BioSystems, USA) on the StepOne System (Bio-Rad, USA). U6 was used as the endogenous control for miR-128-3p and GAPDH for PVT1. Each reaction was performed in triplicates and relative expression of target genes was calculated by the $2^{-\triangle \triangle \mathrm{Ct}}$ method for gene expression in $\mathrm{BC}$ cells and the $2^{-\triangle \mathrm{Ct}}$ method for that in $\mathrm{BC}$ plasma. The following primers were used: PVT1 sense (5'-TGAGAA CTGTCCTTA CGTGACC-3') and antisense (5'-AGA GCACCAAGACTGGCTCT-3'); GAPDH sense (5'-GGG AGCCAAAAGGGTCAT-3') and antisense (5'-GAGTCC TTCC ACGATACCAA-3').

\section{Western blot assay}

Western blot analyses were conducted according to the method described previously [12]. The primary antibodies were: rabbit monoclonal anti-E-cadherin (\#3195, 1:1000, Cell Signaling Technology), rabbit monoclonal anti-Vimentin (\#5741, 1:1000, Cell Signaling Technology), rabbit polyclonal anti-UPF1 (23379-1-AP, 1:1000, ProteinTech), rabbit polyclonal anti-FOXQ1 (23718-1-AP, 1:1000, ProteinTech), mouse monoclonal anti- $\beta$-actin (A5316, 1:5000, Sigma-Aldrich), and mouse monoclonal anti-PCNA (sc-25280, 1:1000, Santa Cruz). The blot signal was detected using ECL (GE Healthcare, UK).

\section{Dual-luciferase reporter assay}

For the PVT1 luciferase reporter assay, pRL-TK-PVT1 or pRL-TK-PVT1 mutant vectors and pGL3 control vector (Promega) were co-transfected into HEK293T along with pSIF-GFP-miR-128-3p precursor plasmid using Lipofectamine ${ }^{\circledR}$ LTX and the Plus reagent (Invitrogen).

For miR-128-3p target gene FOXQ1 luciferase reporter assay, pRL-TK-FOXQ1-3'UTR or pRL-TKFOXQ1-3'UTR mutant vectors and pGL3 control vector (Promega) were co-transfected into cells along with pSIF-GFP-miR-128-3p precursor plasmid using Lipofectamine ${ }^{\circledR}$ LTX and the Plus reagent (Invitrogen). Luciferase activity was detected $48 \mathrm{~h}$ after transfection using the Dual-Glo luciferase reporter assay system (Promega) according to the manufacturer's instructions. Experiments were repeated at least three times.

\section{In vivo experiments}

Female athymic 4-week-old BALB/c nude mice were purchased from HFK Bio-Technology. Co., Ltd (Beijing, China). Hs578t $\left(5 \times 10^{6}\right.$ cells) were injected into the mammary fat pad. Tumor volumes were analyzed using the formula $V=$ length $\times$ width $^{2} / 2$. When the tumor volumes reached $60 \mathrm{~mm}^{3}$, the mice were randomly divided into a control group $(n=6)$ and a si-PVT1 group $(n=6)$. si-PVT1 or the siRNA control $(20 \mu \mathrm{l}, 5 \mathrm{nM}))$ was directly injected into the implanted tumor twice per week. After 3 weeks, all the nude mice were anesthetized by $1 \%$ pentobarbital sodium $(50 \mathrm{mg} / \mathrm{kg})$ and euthanized through the cervical vertebra acetabular method. The tumors and major organs were fixed in 10\%-buffered formalin and embedded in paraffin for immunohistochemistry or hematoxylin \& eosin (H\&E) staining.

\section{Statistical analysis}

All statistical analyses were carried out using Graphpad Prism 5.0 software. Student's t-test or one-way analysis of variance (ANOVA) were performed for comparisons between groups. $P$ value $<0.05$ indicates statistical significance. ${ }^{*} P<0.05,{ }^{* * *} P<0.01,{ }^{* * * *} P<0.001$. 

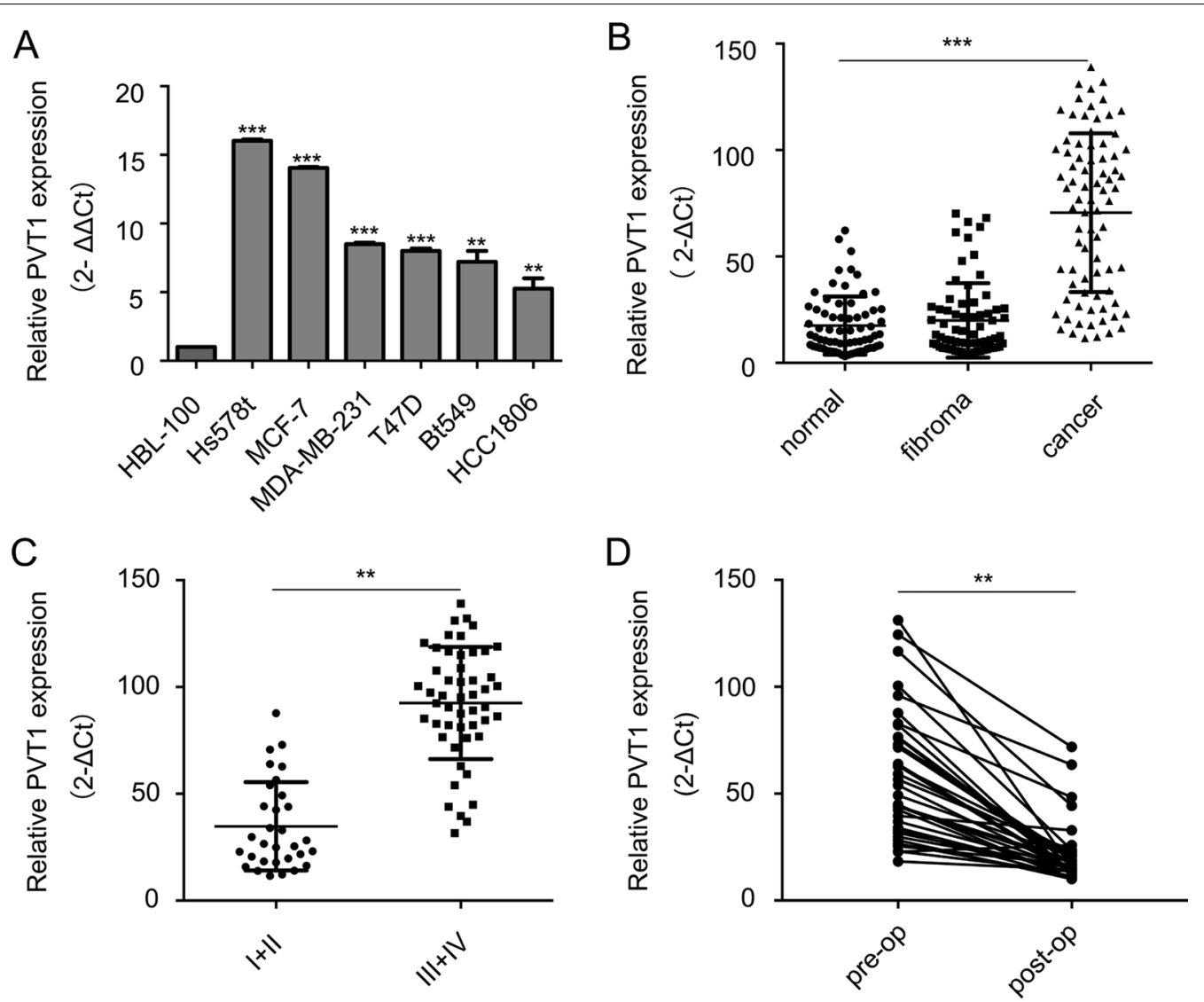

Fig. 1 The expression of PVT1 in BC cell lines and patients' plasma. A The expression levels of PVT1 in BC cell lines and the breast epithelial cell line HBL-100. B The plasma levels of PVT1 in 70 healthy controls, 70 fibroma patients and 80 BC patients. C The plasma levels of PVT1 of BC patients at different stages. D The plasma levels of PVT1 in 35 BC patients with paired preoperative and postoperative specimens. Results were presented as the mean $\pm S D$. ${ }^{* *} p<0.01,{ }^{* * *} P<0.001$

\section{Results}

\section{PVT1 is significantly up-regulated in BC cell lines} and plasma

To explore the expression profile of PVT1 in BC, we first detected PVT1 expression levels in BC cell lines. PVT1 expressions were remarkably higher in BC cells than that in the immortalized mammary epithelial cell line HBL-100 cells (Fig. 1A). Moreover, we tested PVT1 expression levels in plasma samples from $80 \mathrm{BC}$ patients, 70 fibroma patients, and 70 healthy controls. PVT1 levels were significantly increased in BC samples compared with either healthy controls or breast fibroma patients (Fig. 1B). Breast fibromatosis is the most common histologically benign lesion and rarely undergo malignant transformation. The clinical characteristics of the BC patients are listed in Table 1. To further explore the significance of PVT1 expression in $\mathrm{BC}$, we analyzed PVT1 expression in relationship to tumor stages and found that the plasma levels of PVT1 were significantly higher in patients with stage III and
IV than those with stage I and II (Fig. 1C). And we also find the expression of PVT1 has no difference among the subtypes (see Additional file 1: Table). In addition, we analyzed 35 paired preoperative and postoperative plasma samples from BC patients, PVT1 expressions were remarkably reduced in the postoperative samples (Fig. 1D). These results suggested that PVT1 is a candidate predictor for $\mathrm{BC}$ diagnosis and staging assessment. Because the PVT1 expression was the highest in cell line Hs578t and MCF-7, we chose these two lines for further study.

\section{PVT1 promotes BC cell proliferation, migration and invasion in vitro}

To investigate the function consequences of PVT1 overexpression on $\mathrm{BC}$, we introduced three specific si-PVT1s into BC cells. si-PVT1 \#3 was the most efficient in knocking down PVT1 expression (Fig. 2A, see Additional file 1: Figure A). The colony formation assay showed that knockdown of PVT1 significantly inhibited Hs578t and 
Table 1 Association between PVT1 expression and the clinicopathological characteristics of breast cancer patients (all female)

\begin{tabular}{|c|c|c|c|c|}
\hline \multirow[t]{2}{*}{ Characteristics } & \multirow[t]{2}{*}{ Case $(n=80)$} & \multicolumn{2}{|c|}{ Expression of PVT1 } & \multirow[t]{2}{*}{$P$ value } \\
\hline & & $\operatorname{Low}(n=40)$ & High $(n=40)$ & \\
\hline \multicolumn{5}{|l|}{ Age } \\
\hline$\leq 50$ & 35 & 16 & 19 & 0.499 \\
\hline$>50$ & 45 & 24 & 21 & \\
\hline \multicolumn{5}{|l|}{ Tumor size } \\
\hline$\leq 3 \mathrm{~cm}$ & 33 & 11 & 22 & 0.012 \\
\hline$>3 \mathrm{~cm}$ & 47 & 29 & 18 & \\
\hline \multicolumn{5}{|l|}{ TNM stage } \\
\hline$|-| \mid$ & 31 & 21 & 10 & 0.012 \\
\hline||$|-| V$ & 49 & 19 & 30 & \\
\hline \multicolumn{5}{|l|}{ LN metastasis } \\
\hline Yes & 42 & 15 & 27 & 0.007 \\
\hline No & 38 & 25 & 13 & \\
\hline \multicolumn{5}{|l|}{ ER status } \\
\hline Positive & 30 & 13 & 17 & 0.356 \\
\hline Negative & 50 & 27 & 23 & \\
\hline \multicolumn{5}{|l|}{ PRstatus } \\
\hline Positive & 37 & 16 & 21 & 0.361 \\
\hline Negative & 43 & 23 & 20 & \\
\hline \multicolumn{5}{|l|}{ HER2 status } \\
\hline Positive & 36 & 16 & 20 & 0.369 \\
\hline Negative & 44 & 24 & 20 & \\
\hline
\end{tabular}

MCF-7 cell proliferation (Fig. 2B, C). Additionally, the wound healing assay showed that cells transfected with si-PVT1 underwent a slower closing of scratch wound than the control (Fig. 2D, E). Meanwhile, the transwell assay showed that knockdown of PVT1 dramatically decreased Hs578t and MCF-7 cell invasion (Fig. 2F, G). These findings indicate that PVT1 promotes BC cell proliferation, migration and invasion in vitro. We examined protein markers for cancer proliferation, migration and invasion in Hs578t and MCF-7 cells. As shown in Fig. 2H, following the treatment of the si-PVT1, the level of the epithelial marker E-cadherin was upregulated, while that of the mesenchymal marker Vimentin and proliferationrelated protein PCNA was downregulated in $\mathrm{Hs} 578 \mathrm{t}$ and MCF-7 cells. These results indicate that inhibition of PVT1 suppresses cell proliferation, migration, invasion and EMT in BC.

\section{PVT1 promotes BC growth and metastasis in vivo}

To further evaluate the effects of PVT1 in vivo, we injected the si-PVT1 or a negative control intratumorally, when the tumors xenografted with Hs578t cells reached an average of $60 \mathrm{~mm}^{3}$ in immunodeficient mice.
We found that the volumes of tumors in mice treated with the si-PVT1 were markedly smaller than those in mice injected with the control (Fig. 3A, B). Lung and liver samples were obtained to evaluate tumor metastasis, and the number of metastatic cells in liver and lung were significantly reduced by si-PVT1 (Fig. 3C, D). In addition, immunohistochemistry results (Fig. 3E) showed that the expression levels of Ki-67 and vimentin were much lower and that of E-cadherin was higher in tumors treated with si-PVT1 than that with the control. These results implicate that downregulation of PVT1 significantly reduces tumor growth and metastasis in vivo.

\section{PVT1 function as a competing endogenous RNA and regulates FOXQ1 expression by competitively binding miR-128-3p}

It is known that lncRNAs often function as competing endogenous RNAs (ceRNAs) or molecular sponges to recruit microRNAs (miRNAs), regulating their biological functions $[13,14]$. To further explore the mechanism of PVT1 in BC tumorigenesis, we investigated whether miRNAs are involved. By using the online bioinformatics software -starBase (http://starbase.sysu.edu.cn), we observed that PVT1 contains a potential binding site for miR-128-3p (Fig. 4A). As shown in Fig. 4B, miR-128-3p expression in $\mathrm{BC}$ cell lines was lower than that in the immortalized mammary epithelial cell line HBL-100. The plasma level of miR-128-3p was much lower in BC patients than that in fibroma patients and healthy controls (Fig. 4C). We then performed a luciferase reporter assay, in which the Renilla luciferase (Rluc) was upstream of the PVT1 gene in one plasmid and the firefly luciferase as a control in another plasmid; the miR-128-3p binding site in Mut-PVT1 was disrupted. Both plasmids and a miR-128-3p expression plasmid were transfected into 293 T cells. As shown in Fig. 4D, the Rluc activity was significantly reduced with wild-type PVT1 and miR128-3p. However, the Rluc activity with Mut-PVT1 and miR-128-3p showed no statistically significant changes. We next down-regulated PVT1 expression in Hs578t and MCF-7 cells using siRNAs and found PVT1 down-regulation markedly increased miR-128-3p levels (Fig. 4E). Similar results were also observed in xenografted tumors (Fig. 4F).

To identify target genes of miR-128-3p, we used starBase and TargetScan and found a potential miR-128-3p site in the FOXQ1 3'UTR (Fig. 5A). FOXQ1 is a transcription factor involved in the EMT, which is associated with tumor growth and metastasis. A luciferase assay showed that miR-128-3p significantly inhibited reporter activity with the FOXQ1 3'UTR; however, mutation in the putative targeting site in the $3^{\prime} \mathrm{UTR}$ resulted in compete abrogation of the repressive effect (Fig. 5B). 


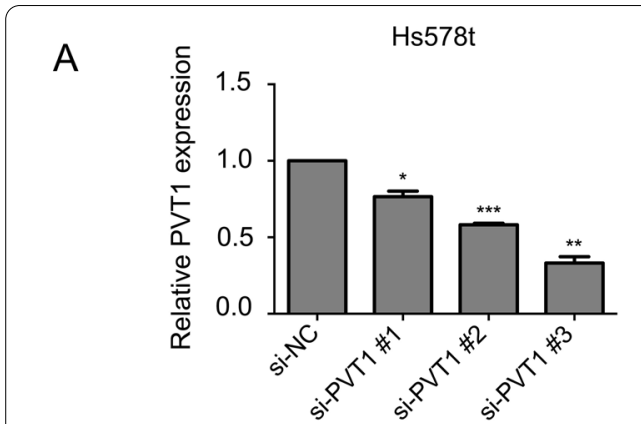

C

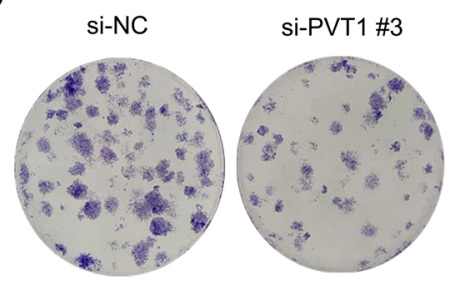

E

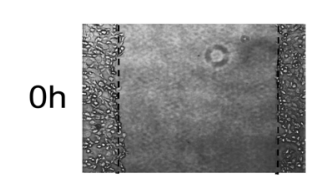

$24 \mathrm{~h}$
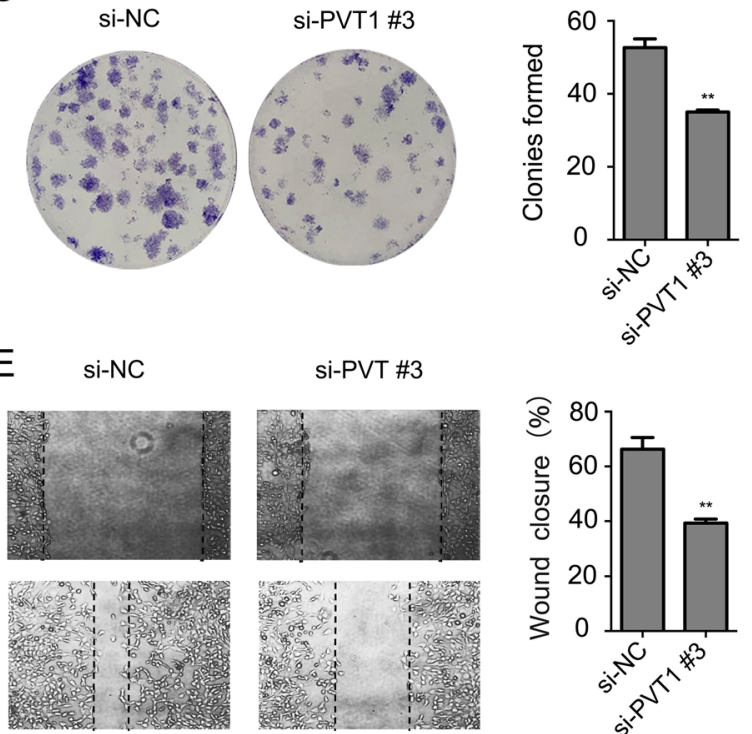

si-PVT \#3

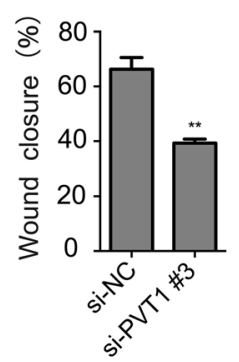

G

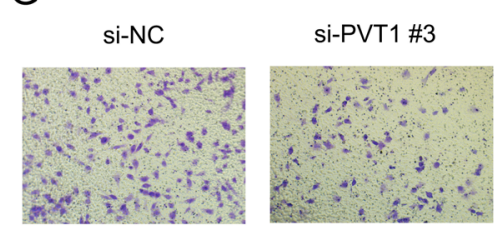

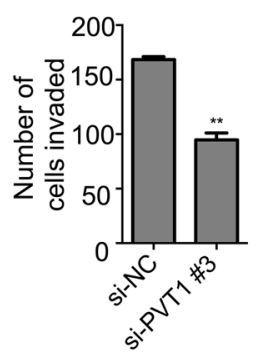

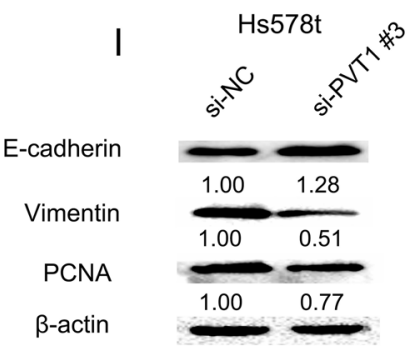

B

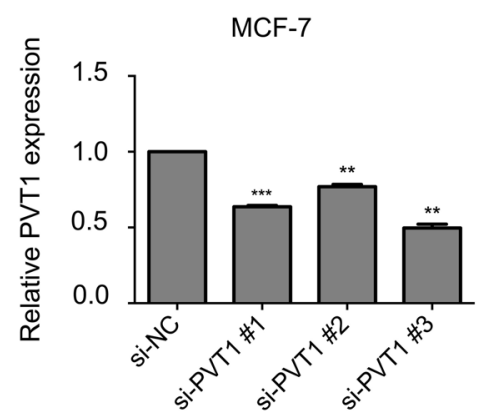

D
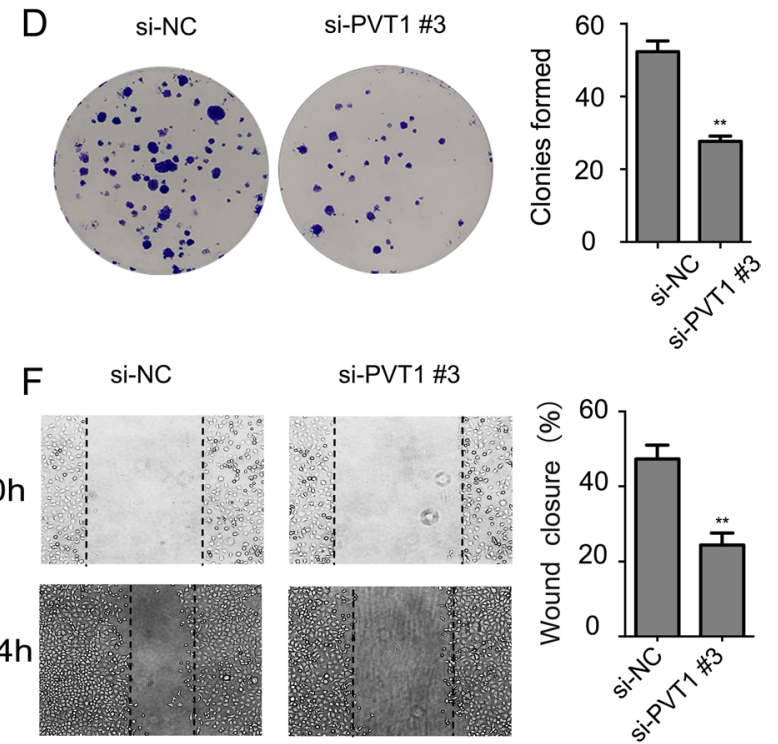

si-PVT1 \#3
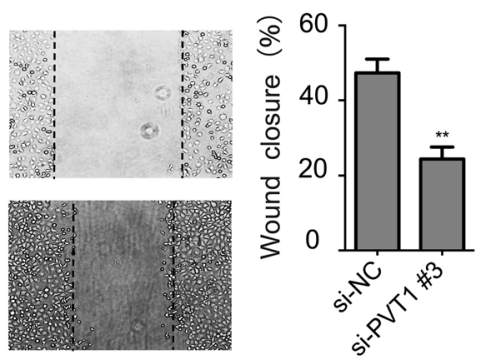

$\mathrm{H}$
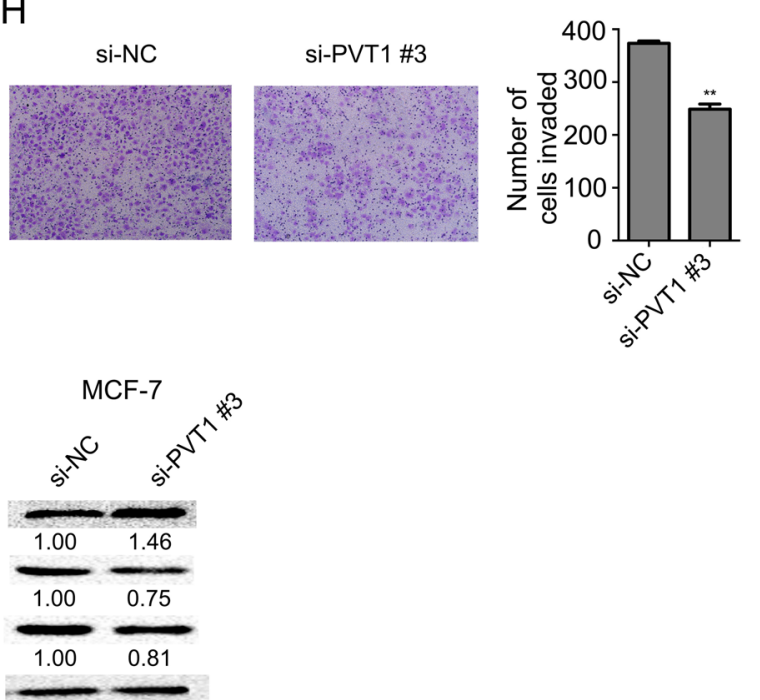

Fig. 2 Inhibition of PVT1 on cell proliferation, migration and invasion of Hs578t and MCF-7 cells. A and B: si-PVT1 knockdown efficiency in Hs578t and MCF-7 cells. C and $\mathbf{D}$ : Colony formation of cells transfected with si-PVT1 or si-NC. $\mathbf{E}$ and $\mathbf{F}$ : Wound healing assays for cell migration. $\mathbf{G}$ and $\mathbf{H}$ : Transwell assays for cell invasion. I: Western blot analyses of proteins that are involved in cell proliferation and metastasis. Results were presented as the mean \pm SD. ${ }^{*} p<0.05,{ }^{* *} p<0.01,{ }^{* *} p<0.001$ 
To examine whether PVT1 regulates FOXQ1 expression, BC cell proliferation and metastasis by directly targeting miR-128-3p, we transfected Hs578t cells with si-PVT1, miR-128-3p inhibitor, or both and evaluated the malignant phenotypes. As shown in Fig. $5 \mathrm{C}$, the colony numbers of Hs578t cells were reduced with siPVT1 transfected, but were increased with miR-128-3p knockdown. Yet with both miR-128-3p and PVT1 knockdown, the colony numbers were lower than that with miR-128-3p knockdown only. Moreover, knockdown of PVT1 markedly inhibited the wound closure rate and reduced cell invasion; inhibition of miR-128-3p exhibited the opposite effects, yet co-transfection with both inhibitors reversed increased migration and invasion of $\mathrm{Hs} 578 \mathrm{t}$ cells mediated by miR-128-3p knockdown (Fig. 5D, E). When PVT1 was knocked down, the protein level of E-cadherin was elevated, whereas PCNA, Vimentin, and FOXQ1 were downregulated. Inhibition of miR-128-3p had opposite effects, and the impact of PVT1 knockdown was partially reversed by co-transfection with both inhibitors (Fig. 5F). These results suggest that PVT1 promotes $\mathrm{BC}$ cell proliferation, migration, and invasion, at least partially, by competitively binding to miR-128-3p.

\section{PVT1 promotes BC proliferation and metastasis by binding UPF1}

To further study the molecular mechanism underlying PVT1's role in BC pathogenesis, we identified proteins potentially associated with PVT1 using starBase. Up-frameshift protein 1 (UPF1), a key factor in nonsense-mediated mRNA decay (NMD), is such a protein. UPF1 was upregulated when PVT1 was knocked down in Hs578t and MCF-7 cells (Fig. 6A). UPF1 antibody pulled down PVT1 in a RIP assay (Fig. 6B). To examine whether PVT1 regulates $\mathrm{BC}$ cell proliferation and metastasis by binding UPF1, we transfected Hs578t cells with si-PVT1\#3, sh-UPF1\#2 (sh-UPF1 \#2 was the most efficient in knocking down UPF1 expression in Hs578t and MCF-7 cells, see Additional file 1: Figure B), or both, and evaluated the malignant phenotypes. As shown in Fig. 6C, PVT1 knockdown reduced colony formation of Hs578t cells, while UPF1 knockdown increased it. Yet inhibition of both UPF1 and PVT1 reversed the increase in colony formation mediated by UPF1 knockdown. Similar results were observed for migration and invasion (Fig. 6D, E). PVT1 knockdown upregulated E-cadherin, but down-regulated PCNA and vimentin. UPF1 knockdown had opposite effects, and the impact of PVT1 knockdown was partially reversed by concurrent UPF1 knockdown (Fig. 6F). These results suggest that PVT1 promotes $\mathrm{BC}$ cell proliferation, migration, and invasion by binding UPF1.

\section{Discussion}

With the development of sequencing technology, many non-coding transcripts have been discovered. Among them, lncRNAs have attracted more and more attention given their wide range of functions. Increasing evidence indicates that abnormal expression of lncRNAs is critical for the development of cancers, including BC. PVT1 was reported to regulate cell proliferation and tumor growth in triple-negative $\mathrm{BC}$ through KLF5/ beta-catenin signaling [15]. In this study, we revealed that the oncogene lncRNA PVT1 plays a vital role in BC progression. We demonstrated that PVT1 was significantly upregulated in $\mathrm{BC}$ patients' plasma, and its high levels in the circulation was correlated with poor prognosis. We further showed that PVT1 promoted $\mathrm{BC}$ cell proliferation, migration and invasion. Mechanistically, we found that PVT1 increased FOXQ1 expression via its sponge activity of miR-128-3p. In addition, PVT1 regulated UPF1 expression by directly binding it, providing added evidence to support PVT1 as an oncogene in $\mathrm{BC}$.

Many lncRNAs function as ceRNAs or molecular sponges to recruit miRNAs, regulating their biological functions. Here we found that PVT1 upregulated FOXQ1 by competitively binding to miR-128-3p, thereby promoting BC cell proliferation and migration. miR-128-3p was upregulated by PVT1 knockdown, which also lead to repression of FOXQ1, a target gene of miR-128-3p. Therefore, the effect of PVT1 on BC cell proliferation and migration could be explained in part by its function as a molecular sponge for miR-128-3p. miR-128-3p exhibits a tumor suppressor role in human malignancies [16]. We found that miR-128-3p was significantly downregulated in BC. FOXQ1, a member of the human Forkhead-Box (Fox) gene family that consists of at least 43 members, induces EMT though repressing E-cadherin expression by targeting the E-box in its promoter region [17]. Higher expression of FOXQ1 was found in multiple cancers, including BC, suggesting that FOXQ1 may play an essential role associated with EMT [18]. Our findings suggest that PVT1 may promote BC tumorigenesis by acting as

(See figure on next page.)

Fig. 3 Downregulation of PVT1 reduces tumor growth and metastasis in vivo. A Tumor tissues from nude mice treated with si-NC and si-PVT1 ( $n=6$ for each group). B Tumor volumes in two groups were evaluated. $\mathbf{C}$ Lung and liver tissues were obtained, and the metastatic cells were visualized. D The incidence of lung and liver metastasis in mice was shown in the table. $\mathbf{E} \| \mathrm{HC}$ analyses of Ki-67, E-cadherin, and vimentin in xenografted tumors 

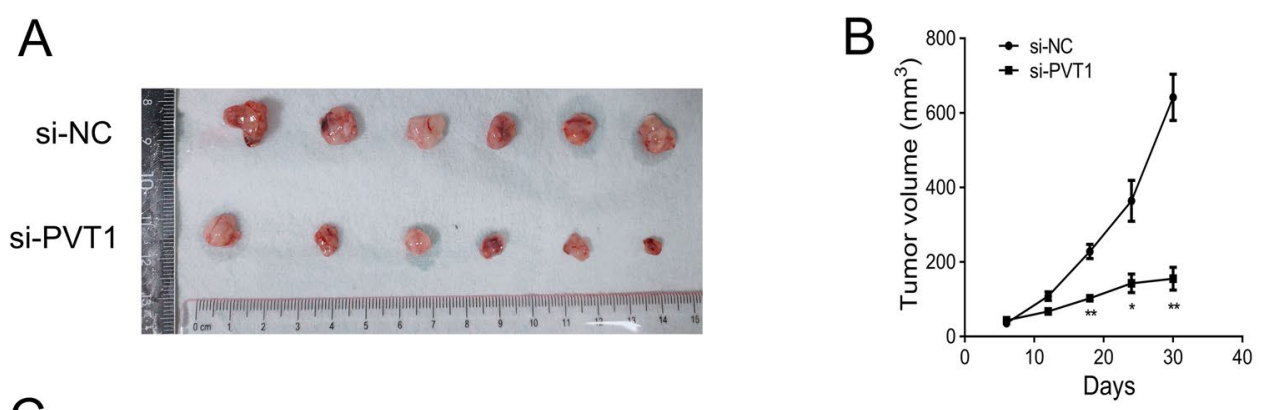

C
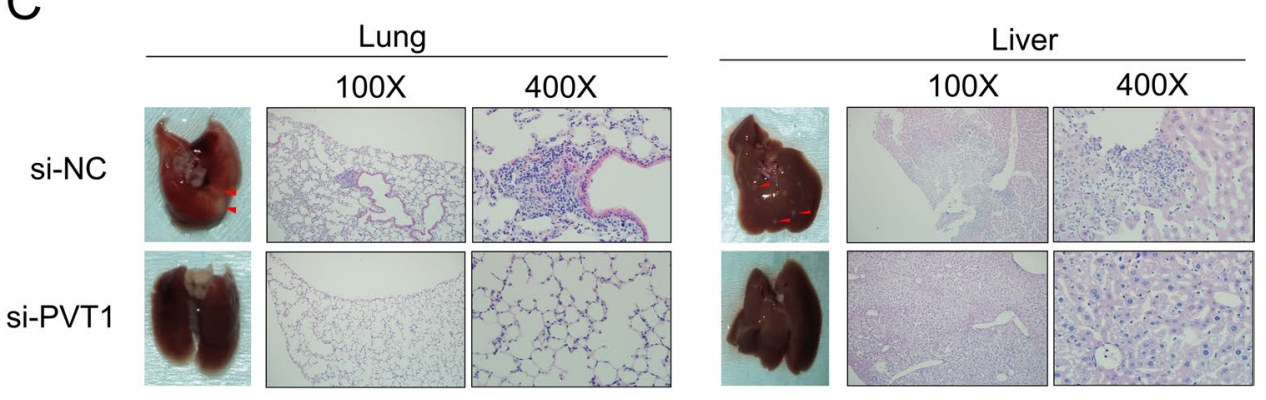

D

\begin{tabular}{lll}
\multicolumn{3}{c}{ Incidence of lung metastasis } \\
\hline Group & Metastasis & $P$ value \\
\hline si-NC & $5 / 6$
\end{tabular}

\begin{tabular}{lll}
\multicolumn{3}{l}{ Incidence of liver metastasis } \\
\hline Group & Metastasis & $P$ value \\
\hline si-NC & $3 / 6$
\end{tabular}

\begin{tabular}{lll} 
si-PVT1 & $1 / 6 \quad 0.021$ \\
\hline
\end{tabular}

si-PVT1 0/6

0.046

E
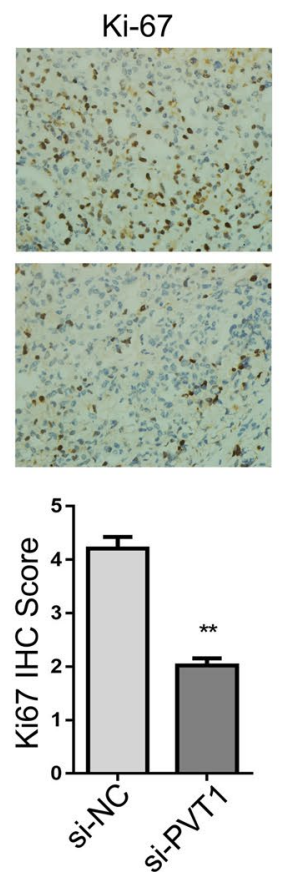

E-cadherin
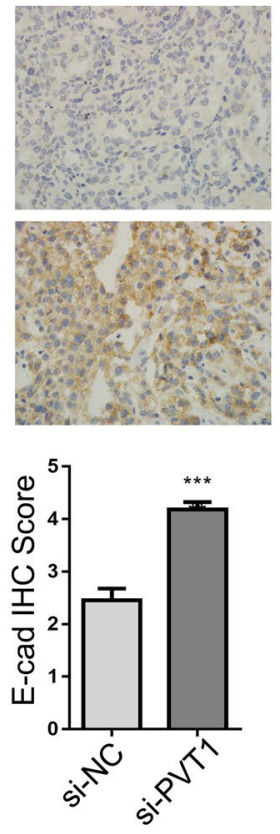

Vimentin
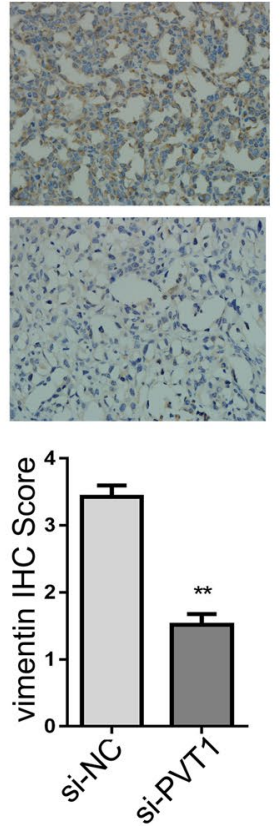

Fig. 3 (See legend on previous page.) 

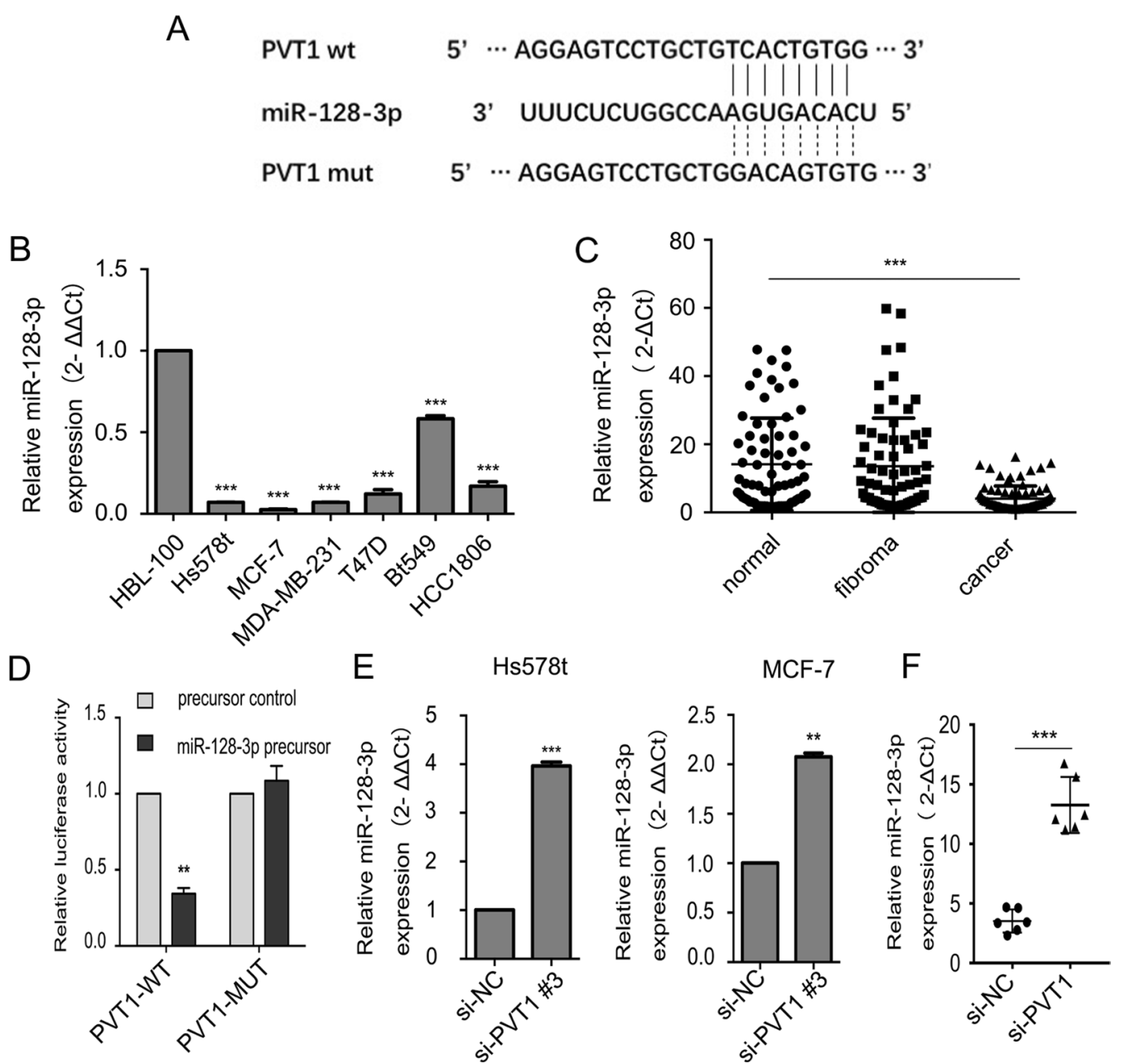

Fig. 4 miR-128-3p was a target of PVT1 in BC cells. A The predicted interaction between PVT1 and miR-128-3p and the mutated miRNA binding site. B The expression levels of miR-128-3p in BC cell lines and the breast epithelial cell line HBL-100. C The plasma levels of miR-128-3p in 70 healthy controls, 70 fibroma patients, and 80 BC patients. D Luciferase reporter assays in $293 \mathrm{~T}$ cells. E The expression levels of miR-128-3p in Hs578t and MCF-7 cells with PVT1 knockdown. F The expression levels of miR-128-3p of tumor tissues from nude mice treated with si-NC and si-PVT1. Results were presented as the mean $\pm S D .{ }^{* *} p<0.01,{ }^{* *} p<0.001$

a ceRNA that competitively binds to miR-128-3p and upregulates FOXQ1 expression.

lncRNAs often to specific protein partners directly to influence their activity or localization. UPF1 is an RNAdependent helicase and ATPase that is required for NMD of mRNAs containing premature stop codons and takes part in cancer progression. Liu and colleagues found that UPF1 gene is commonly mutated in Pancreatic adenosquamous carcinoma [19]. Previous studies showed that UPF1 inhibits the hepatocellular carcinoma progression by targeting $\mathrm{MRP} 2 / \mathrm{ABCC} 2$ or long non-coding RNA
UCA1 [20, 21]. Cao and colleagues found that UPF1 may inhibit TGF- $\beta$ signaling by decreased expression of Smad2/3, MIXL1 and SOX17 in lung adenocarcinoma [22]. Increasing evidence has shown that UPF1 is significantly downregulated in several cancers, showing that NMD is attenuated to permit oncogenesis; UPF1 downregulation promotes EMT in cancer cells [20, 22]. In our study, we revealed that PVT1 directly bound to UPF1 and inhibited its expression, thereby promoting $\mathrm{BC}$ cell proliferation, migration and invasion.

In summary, our findings support that PVT1 acts as an oncogene in BC through binding miR-128-3p and UPF1 


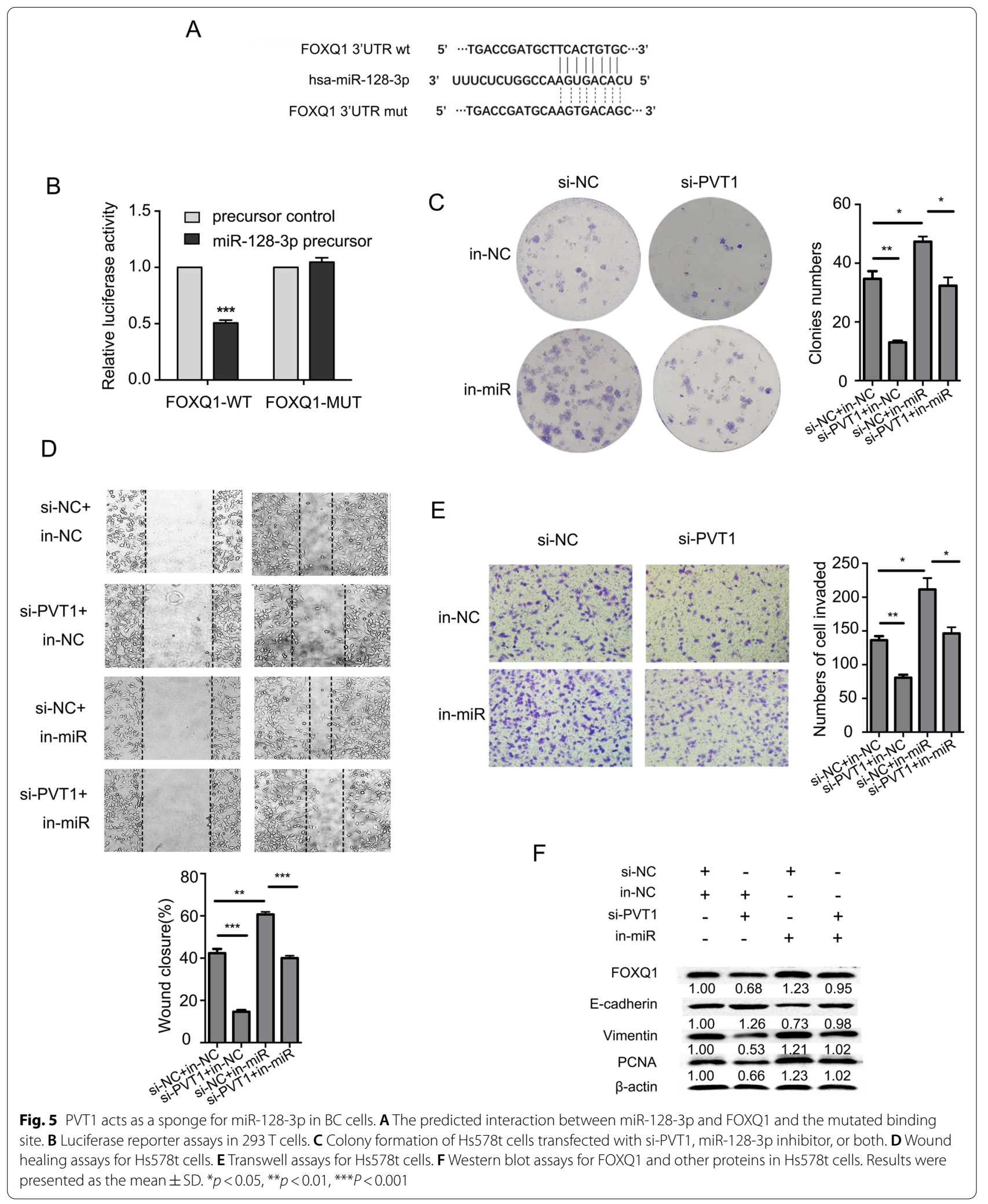




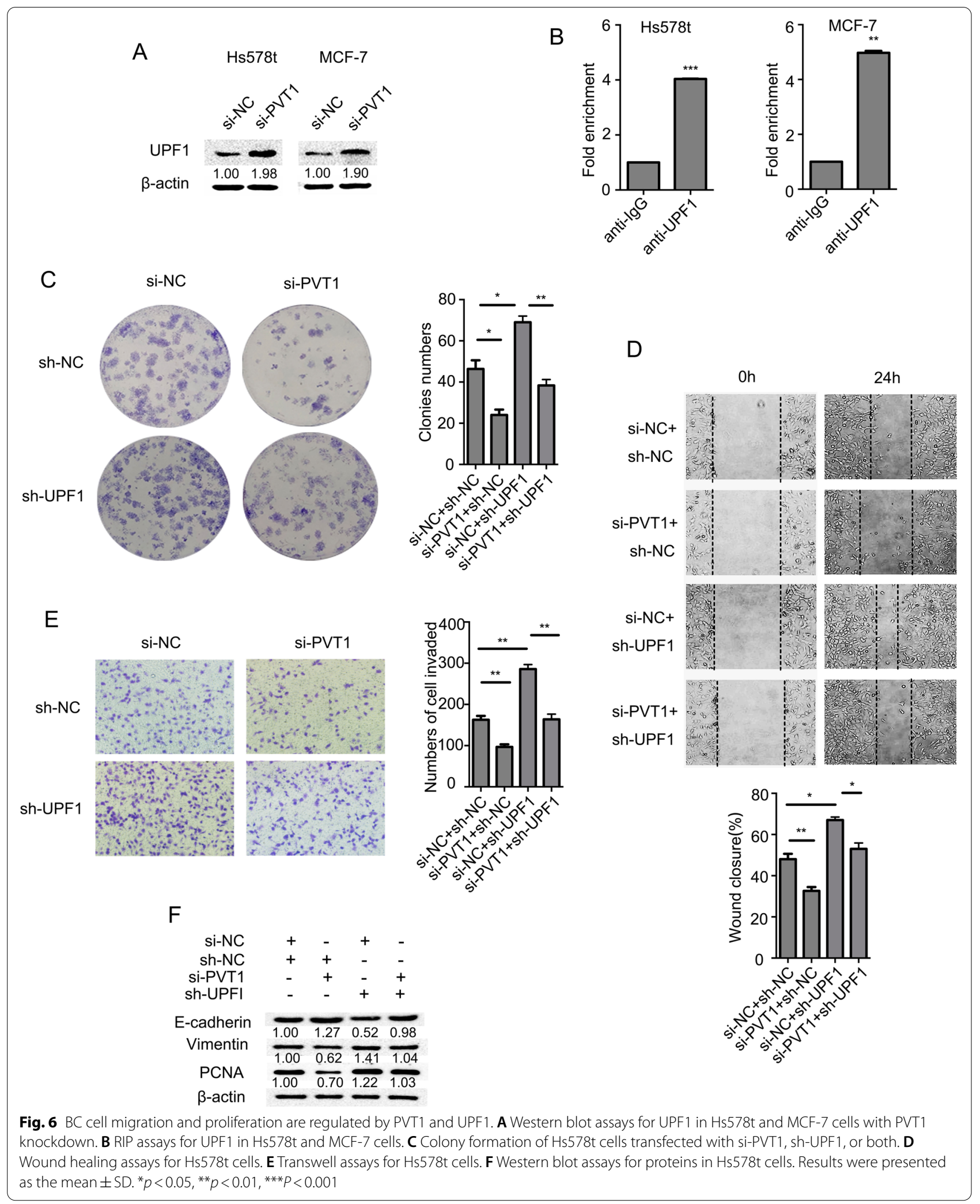




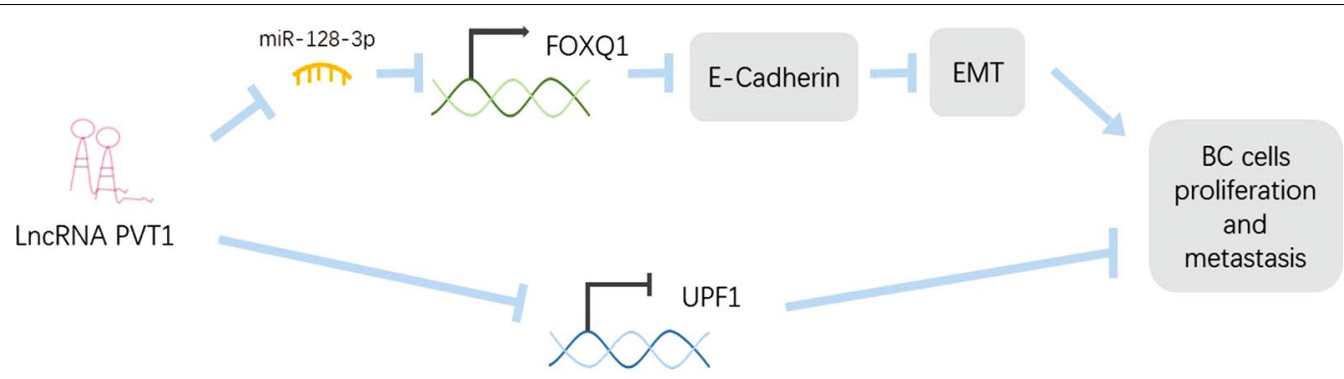

Fig. 7 Schematic model of PVT1 promotes tumor cell proliferation and metastasis in BC

and that PVT1 is a potential target for BC therapeutic development (Fig. 7).

\section{Conclusion}

Taken together, the result of this study indicates that PVT1 functions as an oncogene in breast cancer. High PVT1 expression is associated with tumor progression and poor prognosis. PVT1 promotes breast cancer proliferation and metastasis. As direct targets of PVT1, UPF1 and miR-128-3p mediate the roles of PVT1 in tumor proliferation and metastasis. The effect of PVT1 on breast cancer progression suggests that PVT1 has potential use in antitumor therapies and deserves further investigation.

\section{Abbreviations}

BC: Breast cancer; NPC: Nasopharyngeal carcinoma; PVT1: Plasmacytoma variant translocation 1; UPF1: Up-frameshift protein 1; FOXQ1: Forkhead box Q1; IncRNAs: Long noncoding RNAs; miRNAs: MicroRNAs; ceRNAs: Competitive endogenous RNAs; EMT: Epithelial-mesenchymal transition; siRNA: Small interfering RNA; shRNA: Small hairpin RNA; EDTA: Ethylene diamine tetraacetic acid; PBS: Phosphate-buffered saline; UTR: Untranslated region; qRT-PCR: Quantitative real-time polymerase chain reaction; RIP: RNA immunoprecipitation; IHC: Immunohistochemistry.

\section{Supplementary Information}

The online version contains supplementary material available at https://doi. org/10.1186/s13058-021-01491-y.

Additional file 1: table: Association between PVT1 expression and the subtypes of breast cancer patients (all female). Figure: si-PVT1 and shUPF1 knockdown efficiency in Hs578t and MCF-7 cells. (A) Western blot assays for FOXQ1 and UPF1 in Hs578t and MCF-7 cells. (B) Western blot assays for UPF1 in Hs578t and MCF-7 cells.

\section{Acknowledgements}

We would like to thank all laboratory members for their critical discussion of this manuscript.

\section{Authors' contributions}

SL, WC, HH and TZ carried out the molecular biology analysis, participated in the design of the study and the clinical specimen collection, and drafted the manuscript. TW, XL, QK and $\mathrm{HL}$ carried out the clinical specimen collection, participated in the data analysis, and performed the statistical analysis. YL, $H L$ and $\mathrm{ZL}$ conceived of and designed the study, and participated in the data analysis and coordination, and helped to draft the manuscript. All authors read and approved the final manuscript.

\section{Funding}

This project was supported by grants from the Key project of Natural Science Foundation of Hubei Province (2015CFA078), the Yellow Crane Talent Plan Foundation, Research Fund of Wuhan Public Health Bureau (WX19Q14 and WX18Y11).

\section{Availability of data and materials}

All data generated or analyzed during this study are included in this published article.

\section{Declarations}

Ethics approval and consent to participate

The present study was approved by the Ethics and Scientific Committees of the Central Hospital (Wuhan, China) and complied with the Declaration of Helsinki. All procedures involving animal care and use were approved by the Institutional Animal Care and Usage Committee of Huazhong University of Science and Technology, and were in accordance with the National Policy on Use of Laboratory Animals.

\section{Consent for publication}

Not applicable.

\section{Competing interests}

The authors declare that they have no competing interests.

\section{Author details}

${ }^{1}$ Department of Medical Laboratory, The Central Hospital of Wuhan, Tongji Medical College, Huazhong University of Science and Technology, 26 Shengli St., Jiangan District, Wuhan 430014, China. ${ }^{2}$ Cancer Research Institute of Wuhan, The Central Hospital of Wuhan, Tongji Medical College, Huazhong University of Science and Technology, Wuhan 430014, China. ${ }^{3}$ Key Laboratory for Molecular Diagnosis of Hubei Province, The Central Hospital of Wuhan, Tongji Medical College, Huazhong University of Science and Technology, Wuhan 430014, China. ${ }^{4}$ School of Laboratory Medicine, Hubei University of Chinese Medicine, Wuhan 430065, China. ${ }^{5}$ Department of Medicine, Dan L Duncan Comprehensive Cancer Center, Baylor College of Medicine, Houston, TX 77030, USA. ${ }^{6}$ Department of Oncology, The Central Hospital of Wuhan, Tongji Medical College, Huazhong University of Science and Technology, Wuhan 430014, China.

Received: 5 August 2021 Accepted: 29 November 2021 Published online: 18 December 2021 


\section{References}

1. Siegel RL, Miller KD, Jemal A. Cancer statistics, 2019. CA Cancer J Clin. 2019;69(1):7-34.

2. Evans JR, Feng FY, Chinnaiyan AM. The bright side of dark matter: IncRNAs in cancer. J Clin Invest. 2016;126(8):2775-82.

3. Lin C, Yang L. Long noncoding RNA in cancer: wiring signaling circuitry. Trends Cell Biol. 2018;28(4):287-301.

4. Kopp F, Mendell JT. Functional classification and experimental dissection of long noncoding RNAs. Cell. 2018;172(3):393-407.

5. Xiong XD, Ren X, Cai MY, Yang JW, Liu X, Yang JM. Long non-coding RNAs: an emerging powerhouse in the battle between life and death of tumor cells. Drug Resist Updat. 2016;26:28-42.

6. Mishra S, Verma SS, Rai V, Awasthee N, Chava S, Hui KM, et al. Long noncoding RNAs are emerging targets of phytochemicals for cancer and other chronic diseases. Cell Mol Life Sci. 2019;76(10):1947-66.

7. Cory S, Graham M, Webb E, Corcoran L, Adams JM. Variant $(6 ; 15)$ translocations in murine plasmacytomas involve a chromosome 15 locus at least $72 \mathrm{~kb}$ from the c-myc oncogene. EMBO J. 1985;4(3):675-81.

8. Wang D, Hu Y. Long non-coding RNA PVT1 competitively binds MicroRNA-424-5p to regulate CARM1 in radiosensitivity of non-small-cell lung cancer. Mol Ther Nucl Acids. 2019;16:130-40.

9. Chen J, Yu Y, Li H, Hu Q, Chen X, He Y, et al. Long non-coding RNA PVT1 promotes tumor progression by regulating the miR-143/HK2 axis in gallbladder cancer. Mol Cancer. 2019; 18(1).

10. Zhao J, Du P, Cui P, Qin Y, Hu C, Wu J, et al. LncRNA PVT1 promotes angiogenesis via activating the STAT3/NEGFA axis in gastric cancer. Oncogene. 2018;37(30):4094-109.

11. He Y, Jing Y, Wei F, Tang Y, Yang L, Luo J, et al. Long non-coding RNA PVT1 predicts poor prognosis and induces radioresistance by regulating DNA repair and cell apoptosis in nasopharyngeal carcinoma. Cell Death Dis. 2018;9(2):235

12. Wu T, Chen W, Kong D, Li X, Lu H, Liu S, et al. miR-25 targets the modulator of apoptosis 1 gene in lung cancer. Carcinogenesis. 2015;36(8):925-35.

13. Salmena L, Poliseno L, Tay $Y$, Kats L, Pandolfi PP. A ceRNA hypothesis: the Rosetta Stone of a hidden RNA language? Cell. 2011;146(3):353-8.

14. Tay $Y$, Kats L, Salmena L, Weiss D, Tan Shen M, Ala U, et al. Codingindependent regulation of the tumor suppressor PTEN by competing endogenous mRNAs. Cell. 2011;147(2):344-57.

15. Tang J, Li Y, Sang Y, Yu B, Lv D, Zhang W, et al. LncRNA PVT1 regulates triple-negative breast cancer through KLF5/beta-catenin signaling. Oncogene. 2018;37(34):4723-34.

16. Zhao J, Li D, Fang L. MiR-128-3p suppresses breast cancer cellular progression via targeting LIMK1. Biomed Pharmacother. 2019;115:108947.

17. Qiao Y, Jiang X, Lee ST, Karuturi RK, Hooi SC, Yu Q. FOXQ1 regulates epithelial-mesenchymal transition in human cancers. Cancer Res. 2011;71(8):3076-86.

18. Li Y, Zhang Y, Yao Z, Li S, Yin Z, Xu M. Forkhead box Q1: a key player in the pathogenesis of tumors (Review). Int J Oncol. 2016;49(1):51-8.

19. Liu C, Karam R, Zhou Y, Su F, Ji Y, Li G, et al. The UPF1 RNA surveillance gene is commonly mutated in pancreatic adenosquamous carcinoma. Nat Med. 2014;20(6):596-8.

20. Zhang $H$, You Y, Zhu Z. The human RNA surveillance factor Up-frameshift 1 inhibits hepatic cancer progression by targeting MRP2/ABCC2. Biomed Pharmacother. 2017;92:365-72.

21. Zhou Y, Li Y, Wang N, Li X, Zheng J, Ge L. UPF1 inhibits the hepatocellular carcinoma progression by targeting long non-coding RNA UCA1. Sci Rep. 2019;9(1):6652

22. Cao L, Qi L, Zhang L, Song W, Yu Y, Xu C, et al. Human nonsense-mediated RNA decay regulates EMT by targeting the TGF- $ß$ signaling pathway in lung adenocarcinoma. Cancer Lett. 2017;403:246-59.

\section{Publisher's Note}

Springer Nature remains neutral with regard to jurisdictional claims in published maps and institutional affiliations.

Ready to submit your research? Choose BMC and benefit from:

- fast, convenient online submission

- thorough peer review by experienced researchers in your field

- rapid publication on acceptance

- support for research data, including large and complex data types

- gold Open Access which fosters wider collaboration and increased citations

- maximum visibility for your research: over $100 \mathrm{M}$ website views per year

At BMC, research is always in progress.

Learn more biomedcentral.com/submissions 